Zagazig J. Agric. Res., Vol. 44 No. (1) 2017

\title{
Horticultural Science
}

http:/www.journals.zu.edu.eg/journalDisplay.aspx?Journalld=1\&queryType=Master

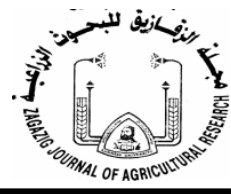

تأثير الرش بمستخلص الثوم وعرق السوس على النمو الخضري لشتلات الكمثرى

محمد عبد الحميد شاكر - وليا عبد الغتي احمد الراوي"

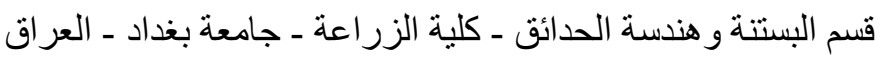

\section{Received: 14/11/2016 ; Accepted: 22/12/2016}

الملخص: أُجريت التجربة في أحد البساتين الخاصة الواقعة في مدينة الغزالية غرب بغداد، للموسمين 2015 و2016،

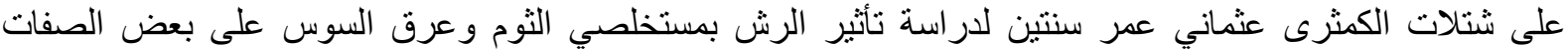

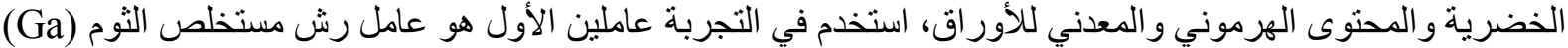

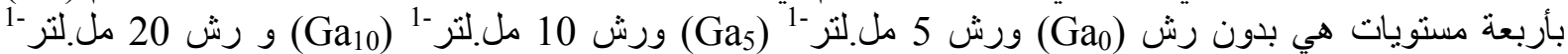

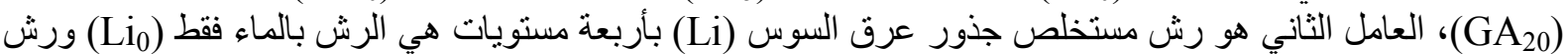

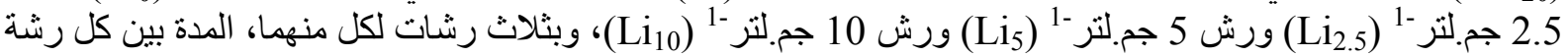
وأخرى 10 أيام، نفذت التجربة وفق نظام تصميم القطاعات العشو ائية الكاملة (RCBD) بثلاثة مكررات وبشتنلثنين لكل

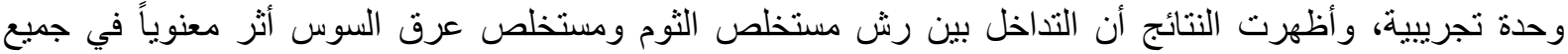

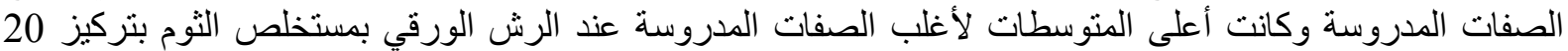

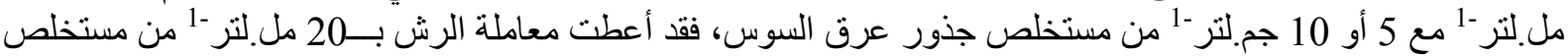

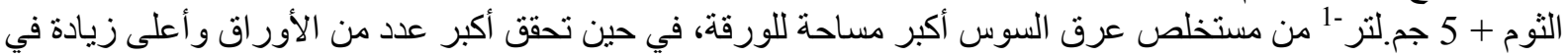
قطر الساق و أعلى محتوى للأور اق من الكلوروفيل نتيجة لمعاملة الرش بمستخلص الثوم ومستخلص عرق السوس بلت بتركيز

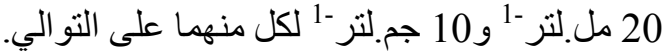

الكلمات الاسترشادية: مستخلص الثوم، مستخلص جذور عرق السوس، مستويات الرش، النمو الخضري، شتلات الكمثرى.

الأمريكية في المرتبة الثانية بعدها إيطاليا وتركيا و أسبانيا

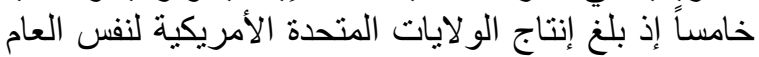

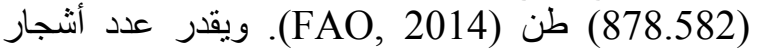
الكمثرى المثمرة في العراق بما يقرب من 547508

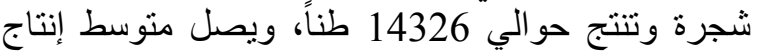

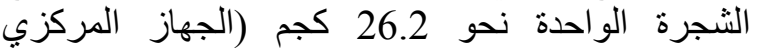
للإحصاء وتكنولوجيا المعلومات، 2013).

في الآونة الأخيرة شاع استعمال المستخلصات الزئات النباتية

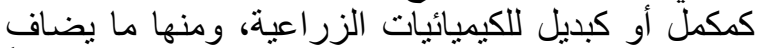

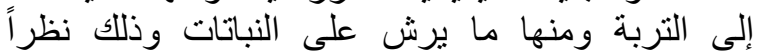

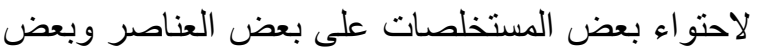
المو اد البديلة لمنظمات النمو ومن بين هذه المستخلصيات

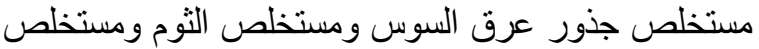

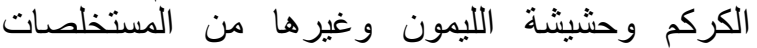

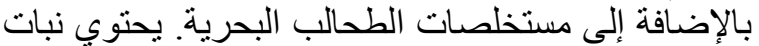

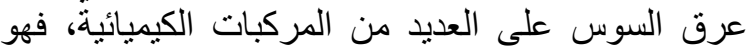
يتميز بوجود مركبات كيميائية ذات مذاق حلو الوبر ، إذ يحتوي

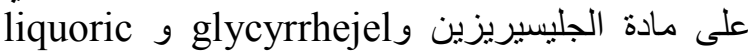
glabrin glabridin ومركبات فلافو نيدية ونهاين acid

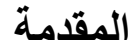

الكمثرى Pyrus communis من الفاكهة متساقطة

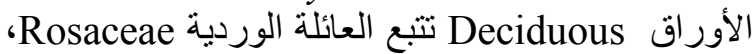

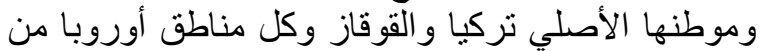

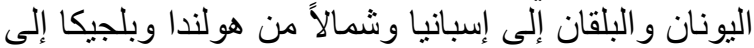

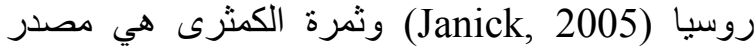

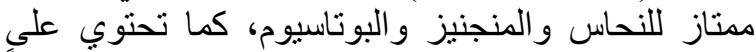

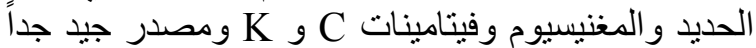
لفيتامين E وكل 100 جم من الثمار يحتوي على ولى 42

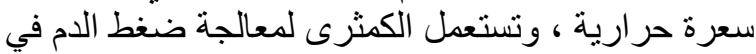

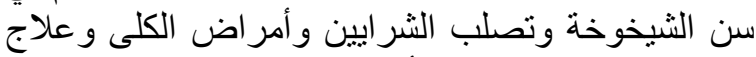

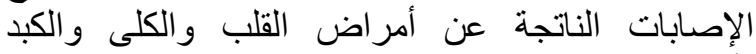
(ألنعيمي ، 2010 ؛ العيسى وبطحة، الإست 2012). بلغ الإنتاج العالمي من الكمثرى عام 2012 حو الي $(23,580,845)$ طن، وبلغت المساحات المزروعة بهات $(1,623,031)$

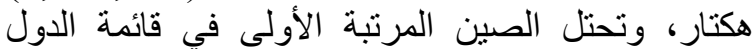

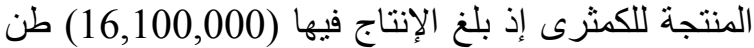
أي ما يزيد عن نصف إنتاج العالم و تأتي الولايات التات المتحدة

*Corresponding author: Tel. :009647717476374

E-mail address: Waleed_dac1@yahoo.com 


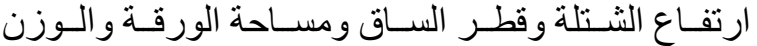

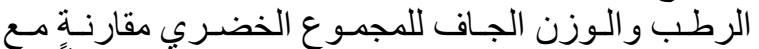

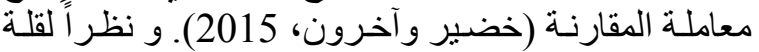

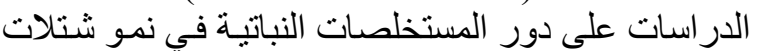

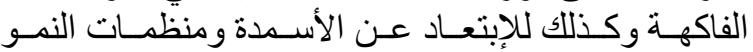

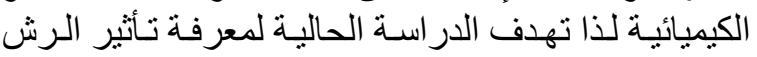

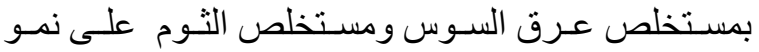
شتلات الكمثرى صنف عثماني.

\section{مواد وطرق البحث}

أُجريت هذه التجربة في أحد البساتين الخاصة الواقعة

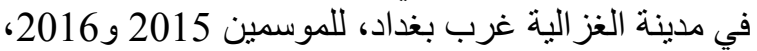

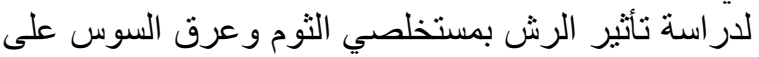

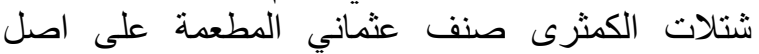

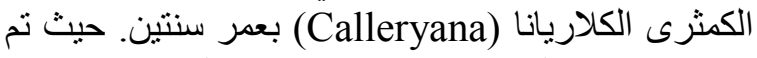

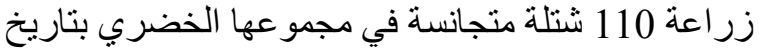
22 / 2 / 2015. رشت الثتنلات حتى البلل الكامل في في

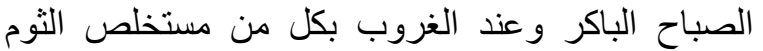

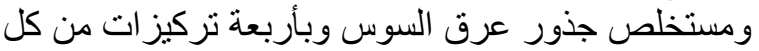

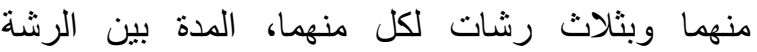

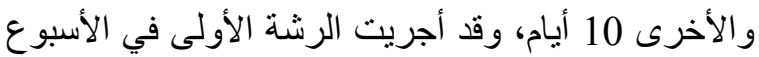

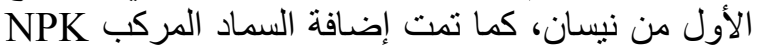

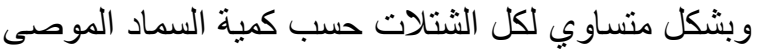
بها. نفذت هذه التجربة على 96 شتلة من شتلات الكمثرى التهى

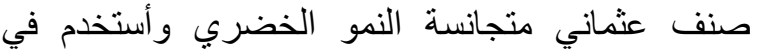

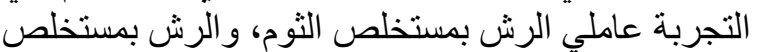
جذور عرق السوس وبذللك تكون المعاملات كالآتي: عامل الرش بمستخلص الثوم ويشمل المعاملات الآتية:

1. بدون رش ( المقارنة) يرمز له بالرمز Ga

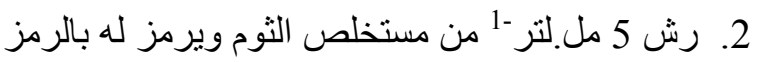
. $\mathrm{Ga}_{5}$

3. رش 10 مل.لتر-1 من مستخلص الثوم ويرمز لله

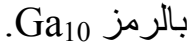

4. رش 20 مل.لتر-1 من مستخلص الثوم ويرمز لله

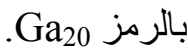

عامل رش مستخلص جذور عرق السوس وتشمل

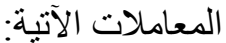

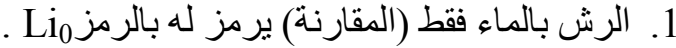

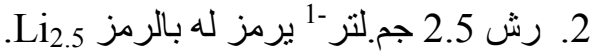
3. رش 5 جم.لتر -1 يرمز له بالرمز 2.

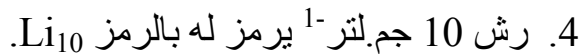

و غير ها (Tyler, 1993) وأن الجليسيريزين وحامضة فئة هما أهم مكونين في عرق السوس إذ لها فيا فعالية مشابهة

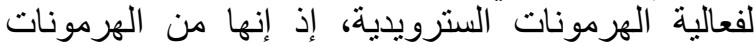

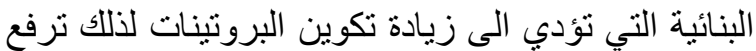

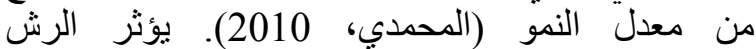

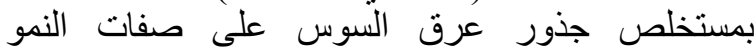
الخضري لأشجار الفاكهة، فقد وجد شئه الكيال العلم (2009) في در استه التي تضمنت رش ثلاثنة نر اكيز من مستخلص الته

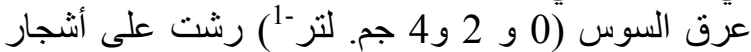

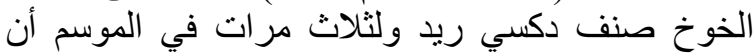
الرش الورقي بمستخلص عرق رق السوس وبت وبتركيز 2

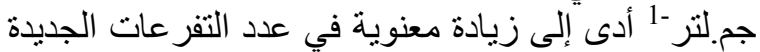

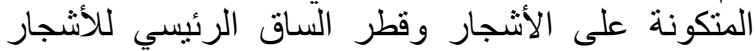

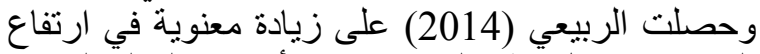

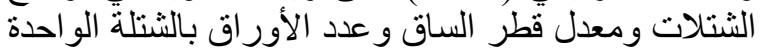

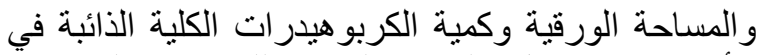

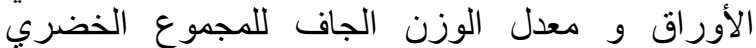

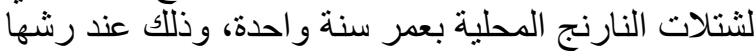

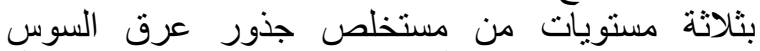

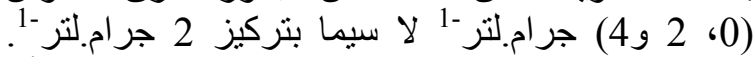
وبين(Al-Hadethi and Al-Kubaisy, 2015) أن رش أشجار الخوخ المبطط Peento بأربعة مستويات من

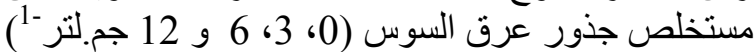

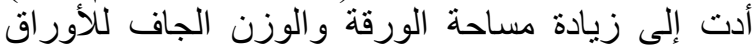

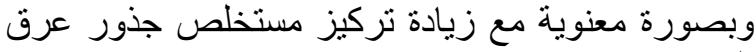

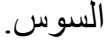

أمسا الثوم فهو نبـات عشبـي و المـو اد الفعالـة فيسه هـي Alina Aliina ، Inuline ، Alcinine

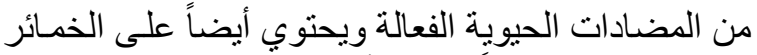

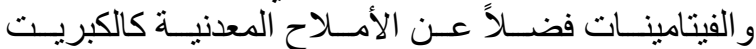

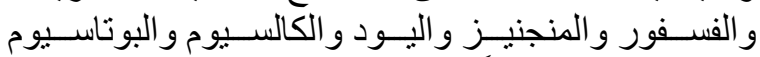

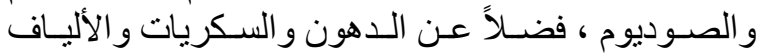

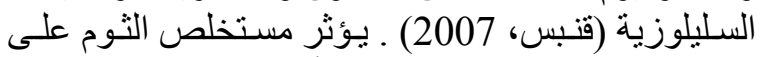
صفات النمو الخضري للعديد من أشجار الفاكهة، فقد ذكر النئ

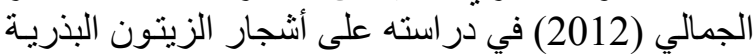

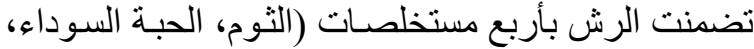

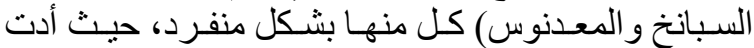

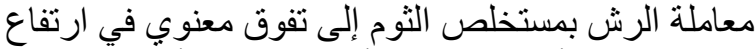

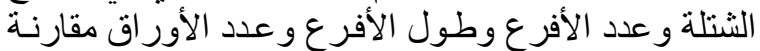
بأثتجار المقارنـة. ووجد (Leonel et al., 2015) في الأني

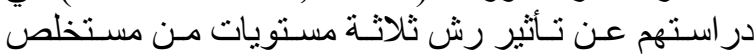

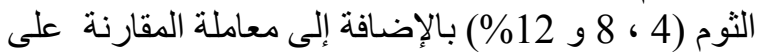

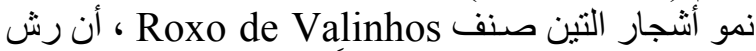

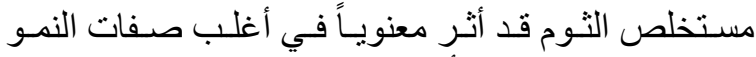

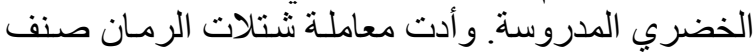

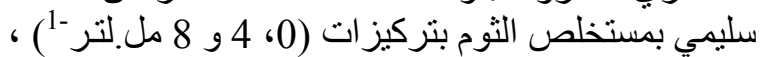

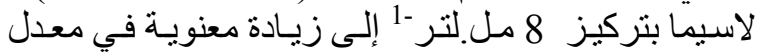


الكلوروفيل الكلي في الأوراق (ملجم.جم-1 وزن غض)

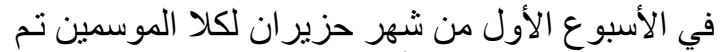
تقدير الكلوروفيل الكلي في أوراق شتيلات الكمثرى، حسب

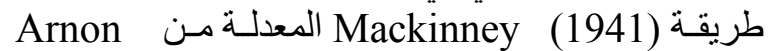

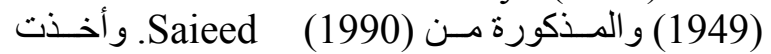

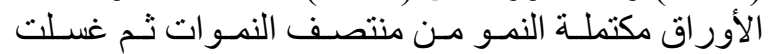

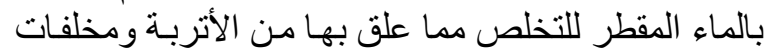

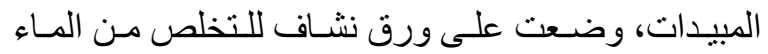

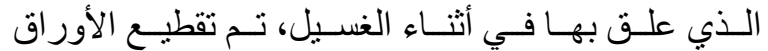

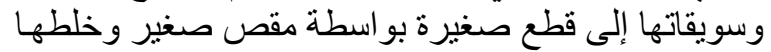

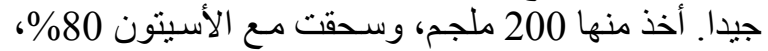

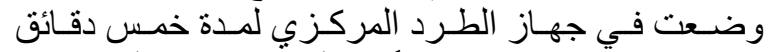

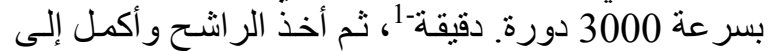

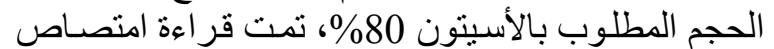

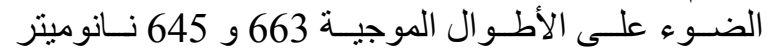

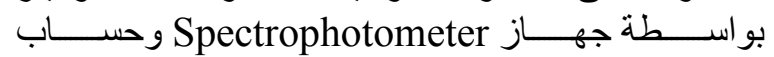
الكلوروفيل الكلي على وفق المعادلة الآتية :

Total Chlorophyll=20.2 A.645 + 8.02 A.663 حيث أن :

A.663 = قـر اءة الجهـاز عــــ الطـول المـوجي 663 نانوميتر.

A.645 = قـر اءة الجهـاز عنـــ الطـول الهـوجي 645 نانوميتر.

\section{النتائج و المناقشة}

\section{مساحة الورقة الواحدة (سم²)}

أنثارت نتائج التحليل الإحصائي في جدول 1 أن الرش التش

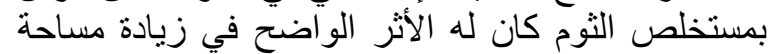
الورقة حيث تفوقت المعاملة الونة

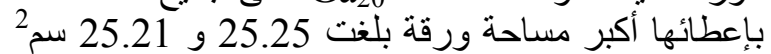
مقارنة بمعاملة المقارنة التي أعطت مساحة ورقة بلغت

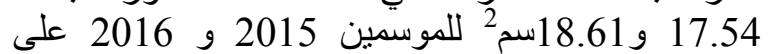

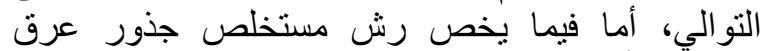

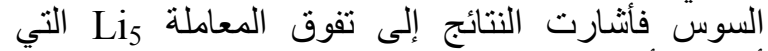
أعطت أعلى مساحة للورقة الواحدة إذ بلغت 27.127 .46

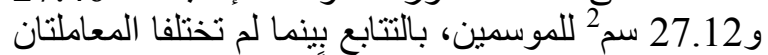
فيما بينهما معنوياً حيث سجلت معاملة المقارنة Li أقل مساحة ورقة بلغت 16.27 و 16.82 سم للموسمين بالتتابع. أما بالنسبة للتنداخل بين العاملين فقد

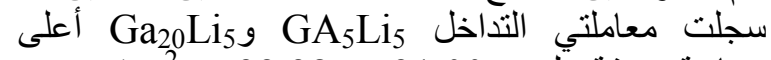

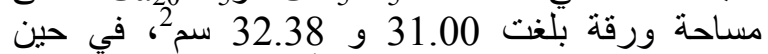
سجلت معاملة المقارنة 13.86 و 14.24 سم2 للموسمين 2015 و 2016 ، على

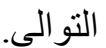

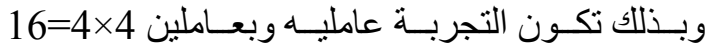
معاملة صمدت وفق تصميم القطاعـات كاملة العشو ائية (RCBD) Randomized Complete Block Design وبثلاثة مكرر ات وبشتلتين للوحدة التجريبية الو احدة. حللت

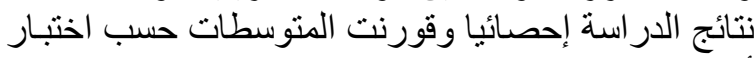
أقل فرق معنوي (LSD) و على مسنوى احتمـال 0.05

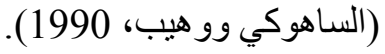

تحضير منقوع الثوم

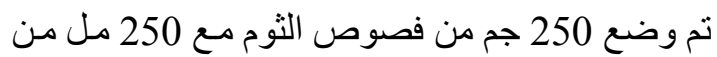
الماء المقطر وخلطت بوساطة خلاط كهربائي وتم ترشيح المحلول الناتج بطبقتين من قماش الململ (الثناش) حيث تم تم

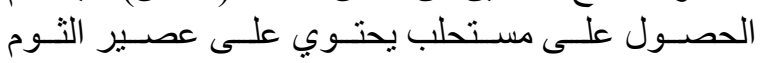

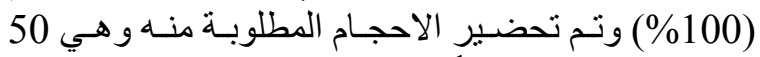
و100 و 200 مل لتنر-1 (العامري، 2001).

\section{تحضير منقوع جذور عرق السوس}

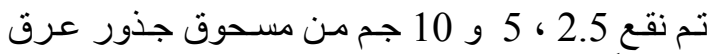

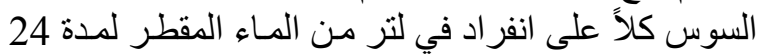

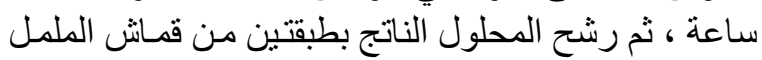

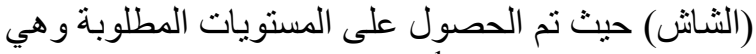

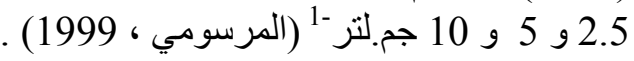

$$
\text { الصفات المدروسة }
$$

\section{مساحة الورقة (سم²)}

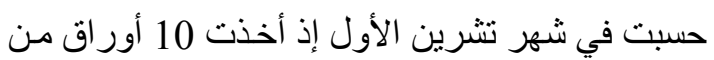
كل نباتات الوحدة التجرييية المقاسة، ووضعت فئ في ماستح

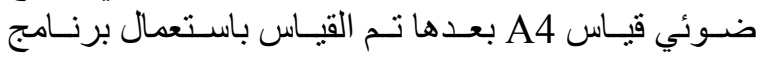

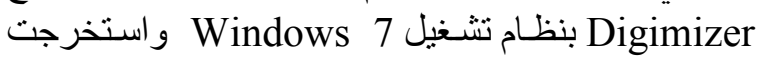
مساحة الورقة الواحدة.

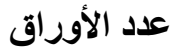

تم حساب عدد الأوراق في نهاية التجربة قبل تساقط

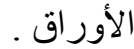

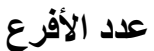

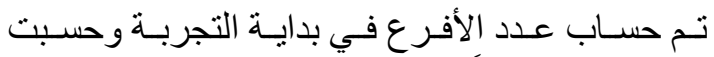

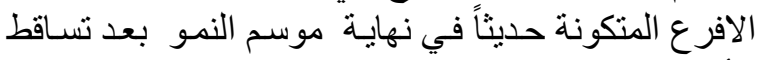
الأوراق، وتم حساب الفرق بينهما و الذي يمثل الزيادة في لئي

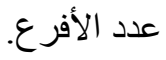

\section{الزيادة في قطر الساق (ملم)}

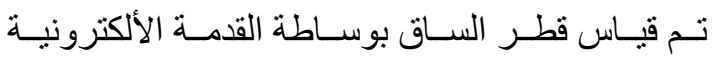

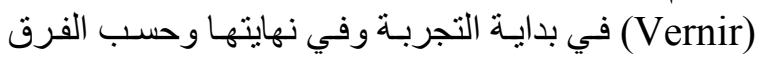
بينهما و الذي مثل الزيادة في قطر الساق في كلا الموسمين. 
جدول1. تأثير رش مستخلص الثوم ومستخلص جذور عرق السوس على مساحة الورقة الواحدة (سم²) لثتلات الكمثرى صنف عثماني للموسمين 2015 و 2016

\begin{tabular}{|c|c|c|c|c|c|c|c|c|c|c|}
\hline \multicolumn{5}{|c|}{2016} & \multicolumn{6}{|c|}{2015} \\
\hline المتوسط & $\mathbf{G a}_{20}$ & $\mathbf{G a}_{10}$ & $\mathrm{Ga}_{5}$ & $\mathbf{G a}_{0}$ & المتوسط & $\mathbf{G a}_{20}$ & $\mathbf{G a}_{10}$ & $\mathbf{G a}_{5}$ & $\mathbf{G a}_{0}$ & \\
\hline 16.82 & 20.32 & 17.40 & 15.30 & 14.24 & 16.27 & 18.82 & 17.22 & 15.17 & 13.86 & $\mathbf{L i}_{0}$ \\
\hline 18.61 & 20.96 & 19.58 & 17.22 & 16.66 & 18.57 & 23.15 & 19.48 & 17.37 & 14.28 & $\mathbf{L i}_{2.5}$ \\
\hline 27.12 & 32.38 & 30.26 & 25.40 & 20.42 & 27.46 & 30.24 & 26.10 & 31.00 & 22.50 & $\mathbf{l i}_{5}$ \\
\hline 25.38 & 27.18 & 26.14 & 25.06 & 23.12 & 24.51 & 28.80 & 25.62 & 24.11 & 19.52 & $\mathbf{L i}_{10}$ \\
\hline \multirow[t]{3}{*}{2.87} & \multicolumn{4}{|c|}{5.74} & 2.13 & \multicolumn{4}{|c|}{4.26} & LSD 5\% \\
\hline & \multirow[t]{2}{*}{25.21} & 23.35 & 20.75 & 18.61 & & 25.25 & 22.11 & 21.91 & 17.54 & \multirow{2}{*}{ المتوسط } \\
\hline & & 2.87 & & & & & 2.13 & & & \\
\hline
\end{tabular}

معاملة المقارنة على بقية المعاملات بإعطائها أعلى عدد

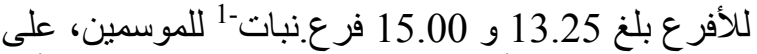

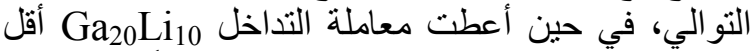
عدد للأفرع و كان 6.50 و 8.00 فرع. التبات فئن للموسمين،

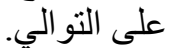

\section{الزيادة في قطر الساق (ملم)}

تبين النتائج في جدول 4 أن معدل الزيادة بقطر الساق

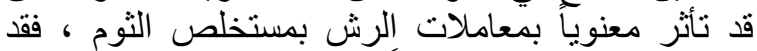

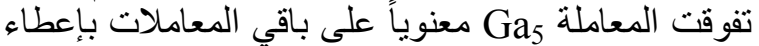
أعلى معدل للزيادة في قطر الساق بلغ بلغ 26.07 و 34.40

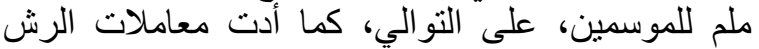

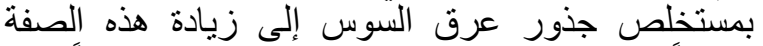

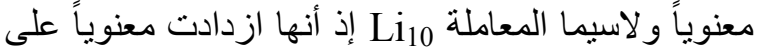

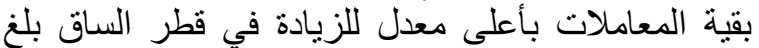

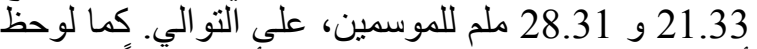

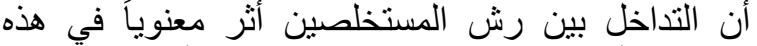

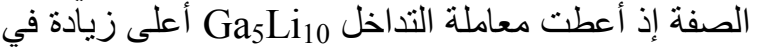

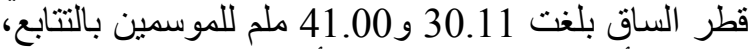
في حين أعطت معاملة المقارنة أقل زيادة في قطر الساق و للموسمين 2015 و 2016 ، بالتتابع.

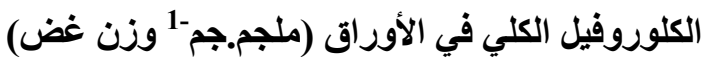

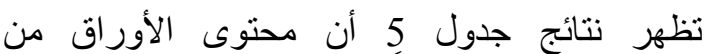

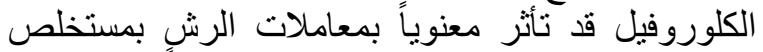

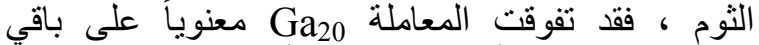

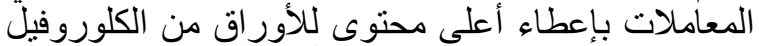

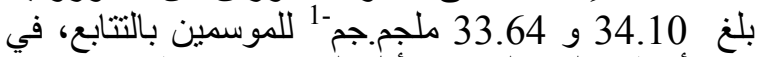
حين أعطت المعاملة 30.97 أقل المعدلات بإعطائها 30.28

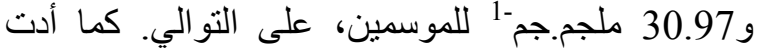

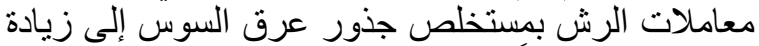

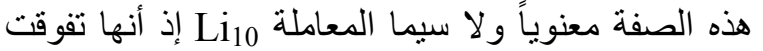

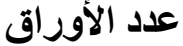

أظهرت النتائج الموضحة في جدول 2 تباين تأثثر

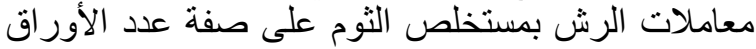
فقد أعطت المعاملة Ga

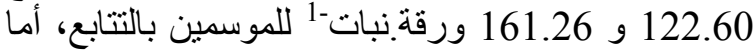
أقل معدل لعدد الأوراق فقد سجل لمعاملة المقارنة 79.62

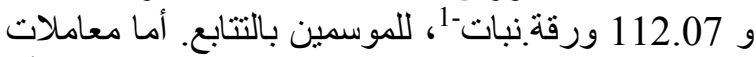

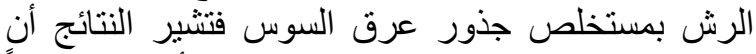

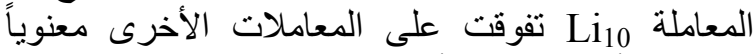
بإعطائها أعلى عدد للأوراق بلغ 115.63 و 154.68

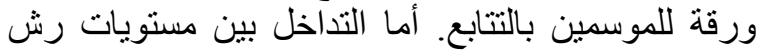
مستخلصي الثوم و عرق السوس فتشير النتائج في جدول 2 إلى أن معاملة التداخل

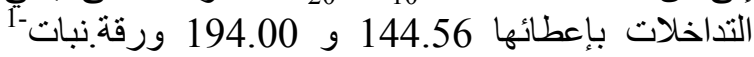
للموسمين بالتتابع، في حين أعطت معان معاملة المقارنة نات $\mathrm{Ga}_{0} \mathrm{Li}_{0}$ نبات-1 للموسمين على التولي.

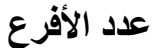

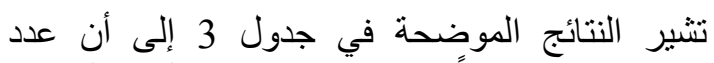
الأفرع انخفض معنوياً مع معاملات رش المش المستخلصين،

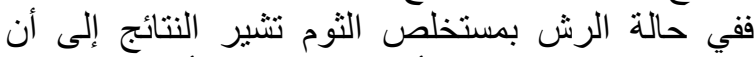
معاملة المقارنة كانت الأعلى في عدد الأفرع 9.63 و و

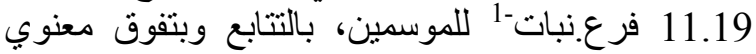

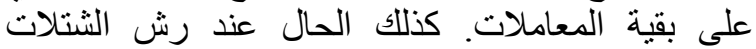
بمستخلص جذور عرق السوس، حيث تثبير النتائج إلى أن الن النيات

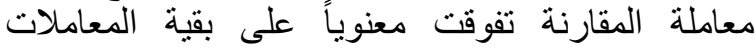
وأعطت أعلى عدد للأفرع بلغ 11.06 و 11.38 فرع.نبات-1 لموسمي الدراسة، على التى التو الي، أما التداخل بين معاملات رش المستخلصات فتنشير النتائج إلى تفوق التي 
جدول 2. تأثير رش مستخلص الثوم ومستخلص جذور عرق السوس على عدد الأوراق لثتلات الكمثرى صنف عثماني للموسمين 2015 و 2016

\begin{tabular}{|c|c|c|c|c|c|c|c|c|c|c|}
\hline \multicolumn{5}{|c|}{2016} & \multicolumn{6}{|c|}{2015} \\
\hline المتوسط & $\mathbf{G a}_{20}$ & $\mathbf{G a}_{10}$ & $\mathbf{G a}_{5}$ & $\mathbf{G a}_{0}$ & المتوسط & $\mathbf{G a}_{20}$ & $\mathbf{G a}_{10}$ & $\mathrm{Ga}_{5}$ & $\mathbf{G a}_{0}$ & \\
\hline 115.44 & 137.24 & 124.16 & 104.78 & 95.56 & 82.51 & 100.27 & 89.88 & 73.78 & 66.12 & $\mathbf{L i}_{0}$ \\
\hline 126.65 & 144.18 & 139.78 & 117.45 & 105.18 & 96.73 & 120.22 & 102.16 & 86.36 & 78.19 & $\mathbf{L i}_{2.5}$ \\
\hline 138.43 & 169.60 & 144.80 & 119.00 & 120.30 & 104.43 & 125.36 & 113.96 & 95.19 & 83.22 & $\mathbf{L i}_{5}$ \\
\hline 154.68 & 194.00 & 162.66 & 134.80 & 127.24 & 115.63 & 144.56 & 124.46 & 102.54 & 90.94 & $\mathbf{L i}_{10}$ \\
\hline \multirow[t]{2}{*}{12.51} & \multicolumn{4}{|c|}{25.02} & 9.67 & \multicolumn{4}{|c|}{19.34} & LSD 5\% \\
\hline & 161.26 & $\begin{array}{c}142.85 \\
12.51\end{array}$ & 119.01 & 112.07 & & 122.60 & $\begin{array}{l}107.62 \\
9.67\end{array}$ & 89.47 & 79.62 & المتوسط \\
\hline
\end{tabular}

جدول 3. تأثثر رش مستخلص الثوم ومستخلص جذور عرق السوس على عدد الأفرع لثتلات الكمثرى صنف عثماني للموسمين 2015 و 2016

\begin{tabular}{|c|c|c|c|c|c|c|c|c|c|c|}
\hline \multicolumn{5}{|c|}{2016} & \multicolumn{6}{|c|}{2015} \\
\hline المتوسط & $\mathbf{G a}_{20}$ & $\overline{G a_{10}}$ & $\overline{\mathrm{Ga}_{5}}$ & $\mathbf{G a}_{0}$ & المتوسط & $\overline{G^{20}}$ & $\overline{G a_{10}}$ & $\overline{\mathbf{G a}_{5}}$ & $\overline{G a_{0}}$ & \\
\hline 12.38 & 11.50 & 10.50 & 12.50 & 15.00 & 11.06 & 10.25 & 9.75 & 11.00 & 13.25 & $\mathbf{L i}_{\mathbf{0}}$ \\
\hline 10.00 & 9.25 & 10.25 & 9.00 & 11.50 & 8.81 & 8.00 & 9.00 & 8.25 & 10.00 & $\mathbf{L i}_{2.5}$ \\
\hline 9.25 & 10.25 & 9.00 & 8.75 & 9.00 & 8.06 & 9.50 & 8.50 & 7.00 & 7.25 & $\mathbf{L i}_{5}$ \\
\hline 9.25 & 8.00 & 8.75 & 11.00 & 9.25 & 7.81 & 6.50 & 7.50 & 9.25 & 8.00 & $\mathbf{L i}_{10}$ \\
\hline \multirow[t]{3}{*}{0.79} & \multicolumn{4}{|c|}{1.58} & 0.54 & \multicolumn{4}{|c|}{1.08} & LSD $5 \%$ \\
\hline & 9.75 & 9.63 & 10.31 & 11.19 & & 8.56 & 8.69 & 8.88 & 9.63 & المته سط \\
\hline & & 0.79 & & & & & 0.54 & & & \\
\hline
\end{tabular}

جدول 4. تأثير رش مستخلص الثوم ومستخلص جذور عرق السوس على الزيادة في قطر الساق (ملم) لثتلات الكمثرى صنف عثماني للموسمين 2015 و 2016

\begin{tabular}{|c|c|c|c|c|c|c|c|c|c|c|}
\hline \multicolumn{5}{|c|}{2016} & \multicolumn{6}{|c|}{2015} \\
\hline المتوسط & $\mathbf{G a}_{20}$ & $\mathrm{Ga}_{10}$ & $\mathrm{Ga}_{5}$ & $\overline{G a_{0}}$ & المتوسط & $\mathbf{G a}_{20}$ & $\mathbf{G a}_{10}$ & $\mathbf{G a}_{5}$ & $\overline{\mathbf{G a}_{0}}$ & \\
\hline 23.87 & 24.80 & 20.38 & 34.57 & 15.71 & 15.67 & 15.18 & 13.27 & 22.56 & 11.68 & $\mathbf{L i}_{0}$ \\
\hline 23.99 & 26.15 & 22.18 & 31.45 & 16.18 & 17.89 & 18.95 & 16.16 & 24.31 & 12.12 & $\mathbf{L i}_{2.5}$ \\
\hline 24.21 & 26.40 & 23.10 & 30.56 & 16.76 & 19.01 & 20.12 & 16.22 & 27.29 & 12.42 & $\mathbf{L i}_{5}$ \\
\hline 28.31 & 29.30 & 25.60 & 41.00 & 17.32 & 21.33 & 23.46 & 18.86 & 30.11 & 12.89 & $\mathbf{L i}_{10}$ \\
\hline \multirow[t]{2}{*}{3.67} & \multicolumn{4}{|c|}{7.34} & 2.02 & \multicolumn{4}{|c|}{4.04} & LSD 5\% \\
\hline & 26.66 & $\begin{array}{c}22.82 \\
3.67\end{array}$ & 34.40 & 16.49 & & 19.43 & $\begin{array}{l}16.13 \\
2.02\end{array}$ & 26.07 & 12.28 & المتوسط \\
\hline
\end{tabular}


جذول 5. تأثير رش مستخلص الثوم ومستخلص جذور عرق السوس على محتوى أوراق شتّلات الكمثرى صنف عثماني

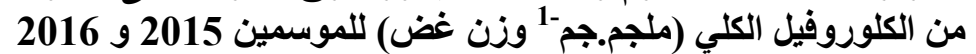

\begin{tabular}{|c|c|c|c|c|c|c|c|c|c|c|}
\hline \multicolumn{5}{|c|}{2016} & \multicolumn{6}{|c|}{2015} \\
\hline المتوسط & $\mathbf{G a}_{20}$ & $\mathbf{G a}_{10}$ & $\mathbf{G a}_{5}$ & $\mathbf{G a}_{0}$ & المتوسط & $\mathbf{G a}_{20}$ & $\mathbf{G a}_{10}$ & $\mathrm{Ga}_{5}$ & $\mathbf{G a}_{0}$ & \\
\hline 31.12 & 31.80 & 31.32 & 30.82 & 30.54 & 30.48 & 31.36 & 30.58 & 30.21 & 29.76 & $\mathbf{L i}_{0}$ \\
\hline 31.75 & 32.90 & 31.88 & 31.26 & 30.94 & 32.06 & 33.90 & 33.29 & 31.15 & 29.88 & $\mathbf{L i}_{2.5}$ \\
\hline 32.89 & 34.36 & 33.48 & 32.56 & 31.16 & 33.08 & 35.01 & 33.80 & 33.14 & 30.37 & $\mathbf{L i}_{5}$ \\
\hline 33.50 & 35.48 & 34.40 & 32.90 & 31.22 & 33.95 & 36.12 & 35.20 & 33.37 & 31.12 & $\mathbf{L i}_{10}$ \\
\hline 0.56 & & & & & 0.83 & & & .66 & & LSD 5\% \\
\hline & 33.64 & $\begin{array}{c}32.77 \\
0.56\end{array}$ & 31.89 & 30.97 & & 34.10 & $\begin{array}{r}33.22 \\
0.83\end{array}$ & 31.97 & 30.28 & المتوسط \\
\hline
\end{tabular}

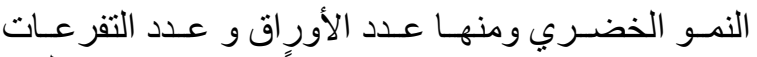

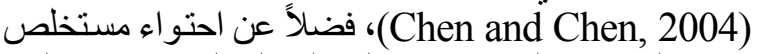

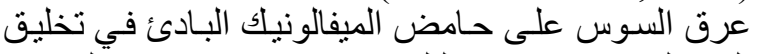

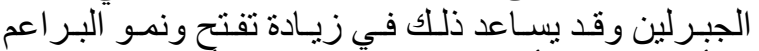

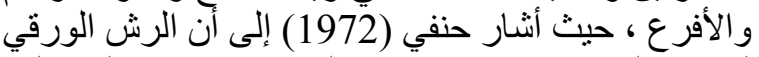

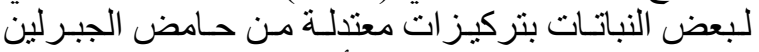

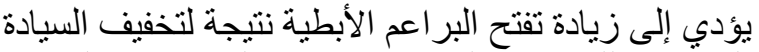

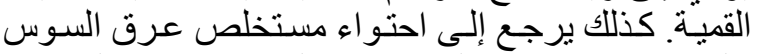

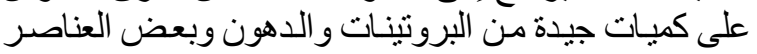

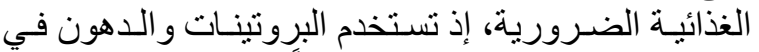

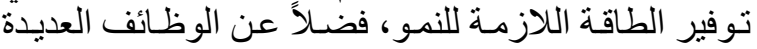

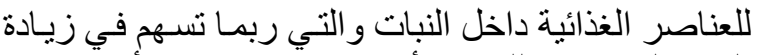

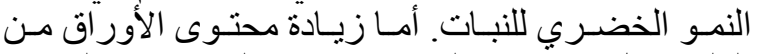

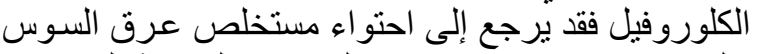

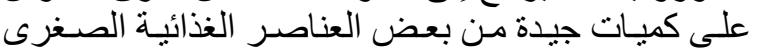

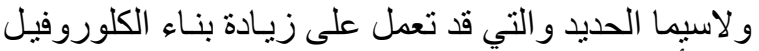
وقلة أكسدته (حداد وبايرلي، 2015 2009) ـ تتفق هذه النتائج مع النع ماوجده (Abd El-Hamied and El-Amary, 2015)

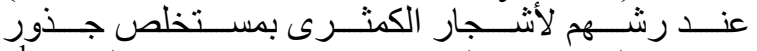

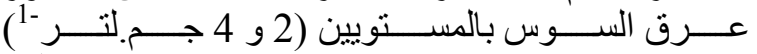
و) (Al-Hadethi and Al-Kubaisy, 2015) على أثنجار الخوخ و) (Mohammed et al., 2016) في

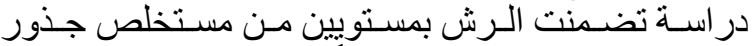
عرق السوس (5 و 10 جم.لتر -1) على شتلات اللوز.

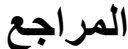

الجمـالي، نغــم محمـود (2012). تـأثنثر معاملــة شـتلات الزيتون Olea europaea L بعناصر كيميائيـة و

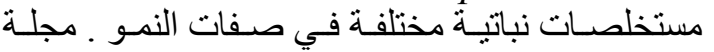
جامعة كربلاء العلمية، 10(3) :90-90

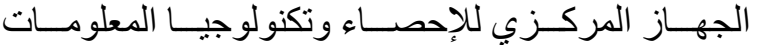

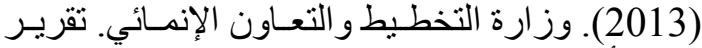

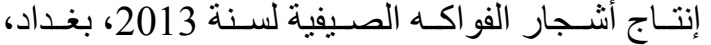

معنويـاً على بقيـة المعاملات بـأعلى محتوى للأور اق مـن

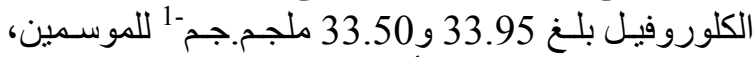

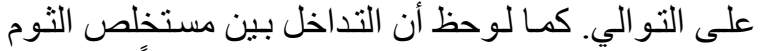

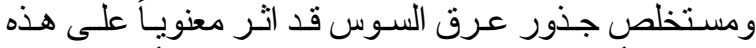

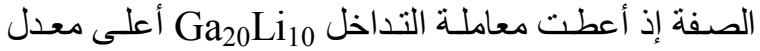

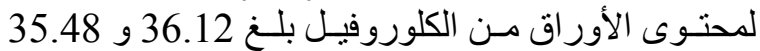

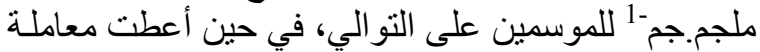

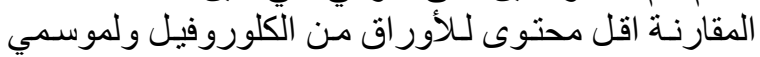

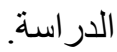

يعود تأثير مستخلص الثوم إلى دور المغذيات الكبرى التئي

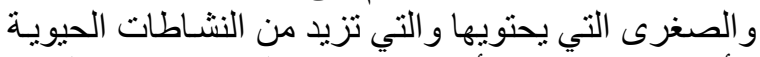

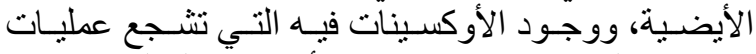

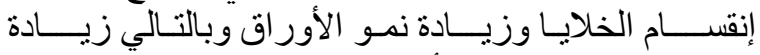

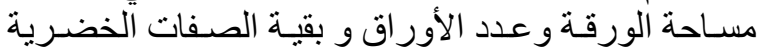
(جacurar and Krejci, 2010)

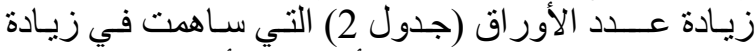
نشاط عملية البناء الضونئي، الأمر الذي أدي أدى إلى استغلانلاد

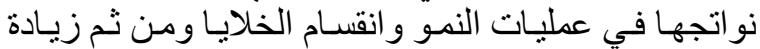

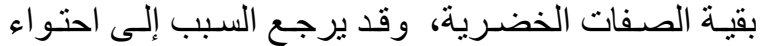

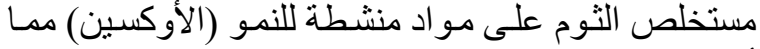

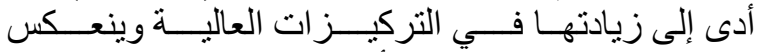

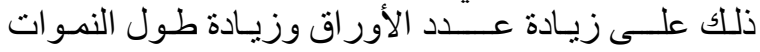

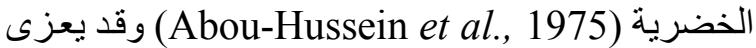
سبب الزيادة في محتوى الأوراق من الكلوروفيل إلى دور

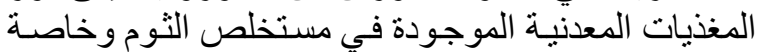

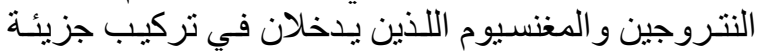
الكلوروفيل (Pacurar and Krejci, 2010). تتفق هذه النتائج مع ما وجده (2014) Armanious (2015) على كرمات العنب و) (Leonel et al., 2015) في در استهم عن تأثير رش ثلاثة مستويات من مستخلص الثوم على أشجار التين.

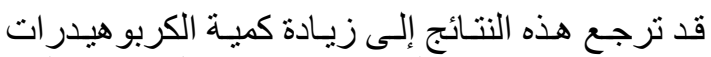

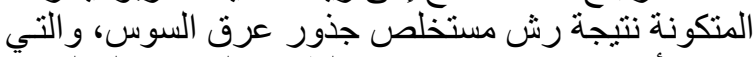

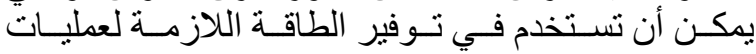


قنبس، أكرم جميل (2007). مستشـار الانسـان في الغذاء

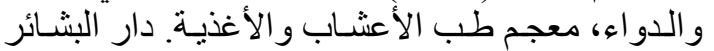

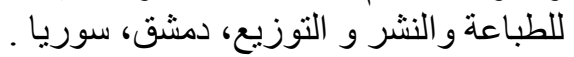

Abd El-Hamied, S.A. and E.I. El-Amary (2015). Improving growth and productivity of "Pear" trees using some natural plants extracts under North Sinai conditions. J. Agric. and Vet. Sci., 8(1):19 .

Abou-Hussein, M.R., S.F. Mostofa and A.Y. Wally (1975). Effect of garlic bulb crude extract on flowering-sex ratio and yield of squash. Egypt. J. Hort., 2 (1): 129-139.

Al-Hadethi, M.E.A and I.M. Al-Kubaisy (2015). Response of Peento peach trees to foliar spray with some plant extracts. Zagazig J. Agric. Res., 42(5): 1029-1034.

Armanious, M.K.U. (2014). The synergistic effect of spraying some plant extracts with some macro and micro nutrients of Thompson Seedless grapevines. Int. J. Plant and Soil Sci., 3 (10): 1290-1301 .

Arnon, D.I. (1949). Copper enzymes isolated chloroplasts polyphenol oxidase in Beta vulgaris . Plant Physiol., 24: 1-15.

Chen, L.S. and L. Chen (2004). Photosynthetic enzymes and carbohydrate metabolism of apple leaves in response to nitrogen limitation. J. Hort. Sci. Biotechnol., 79 (6): 923-929.

FAO (2014). FAO STAT Agricultural statistics database .http://www. Fao. Org.

Janick, J. (2005). The origin of fruits, fruit growing and fruit breeding. Plant Breed.Rev., 25: 255- 320.

Leonel, S., M.A. Tecchio and G.M.D. Cóser (2015). Dormancy breaking of the fig tree with hydrogen cyanamide and garlic extract. British J. Appl. Sci. and Technol., 10 (1): 1-10.

Mackinney, G. (1941). Absorption of light by chlorophyll solution. J. Biol. Chem., 140: 315 - 322.

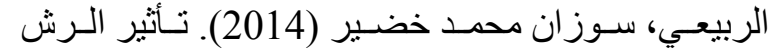

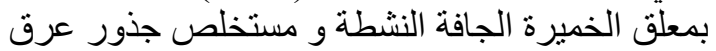
السوس في بعض صفات النمو الخضري النهات و الجذري

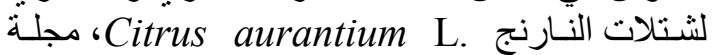

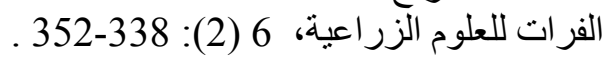

الساهوكي، مدحت مجيد وكريمة و هيب (1990). تطبيقات

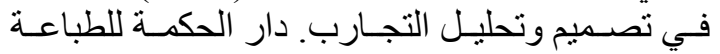
و النشر. الموصل، 488.

العامري ، نبيل جو اد كاظم (2001). تأثثر التغطيس بكل

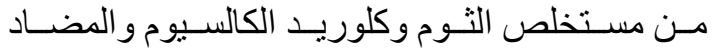

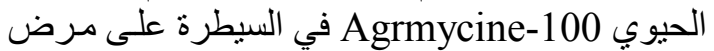

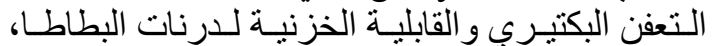
رسالة ماجستير ، كلية الزر اعة، جامعة بغداد.

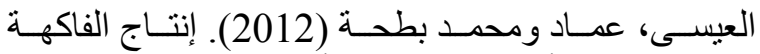

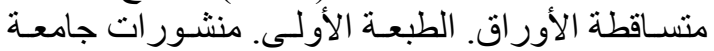

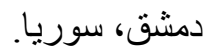

المحمـدي، علـي فـدعم عبـد الله (2010). تـأثير مو اعبـد

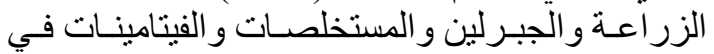

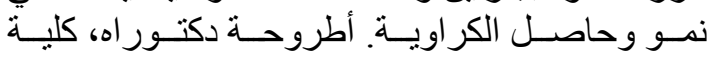
الزر اعة،، جامعة بغداد، العر اق.

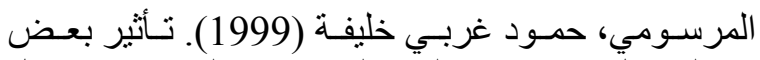

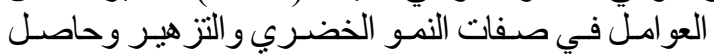

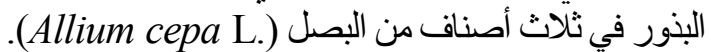
أطروحةٌ دكتور اه، كلية الزر اعة، جامعة بغداد.

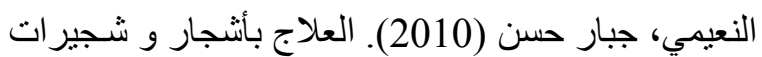

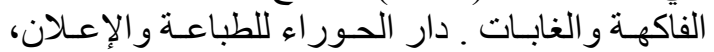

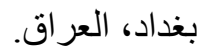

حداد، سهيل ورولا بايرلي (2009). فيزيولوجيا الفاكهة .

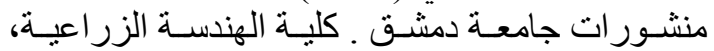

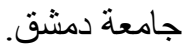

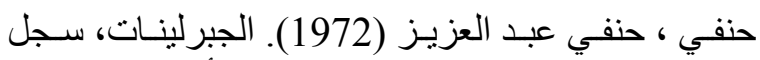

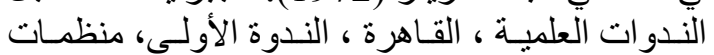

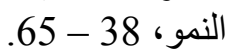

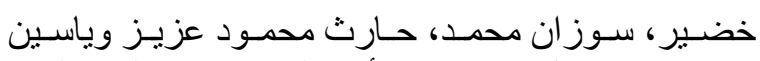

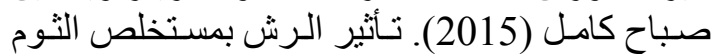

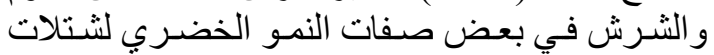

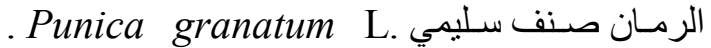
مجلة جامعة كربلاء العلمية ـ 13 (3): 60-65 ـ

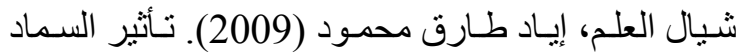
النتروجيني و الرش بحامضي الجبرليك وفيك و الأسكوربيك

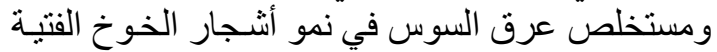

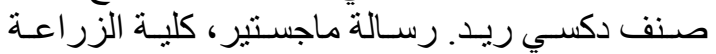
و الغابات، جامعة الموصل، العراق. 
Saieed, N.T. (1990). Studies of variation in primary productivity growth and morphology in relation to the selective improvement of broad-leaved trees species. Ph. D. Thesis Nat. UnivIrland.

Tyler, V. (1993). The Honest Herbal, Pharmaceutical products. Press, Ny., 197 -990 .
Mohammed, T.S., E.K. Nabila, M.S. Abou Rayya and R.A. Eisa (2016). Response of Nonpareil seedlings almond to foliar application of liquorice root extract and bread yeast suspend under South Sinai conditions. J. Innov. in Pharmaceuticals and Biol. Sci., 3 (1): 123-132.

Pacurar, M. and G. Krejci (2010). Garlic consumption and health. Nova Science Publishers, Inc. New York, 1-60.

\title{
EFFECT OF GARLIC AND LICORICE ROOT EXTRACTS SPRAY ON VEGETATIVE GROWTH OF PEAR TRANSPLANTS
}

\author{
Mohammed A. Shakir and W.A.A. Al-Rawi
}

Hort. and Landscape Dept., Coll. Agric., Baghdad Univ., Iraq

\begin{abstract}
This research was conducted in a private orchard in the Ghazaliya city west of Baghdad during 2015/2016 growing seasons to investigate the response of 2 year's old transplants of "Othmani" pear cultivar budded on Calleryana rootstock to foliar application with licorice root extract at four levels $0\left(\mathrm{Li}_{0}\right), 2.5 \mathrm{~g} .1^{-1}\left(\mathrm{Li}_{2.5}\right), 5 \mathrm{~g} .1^{-1}\left(\mathrm{Li}_{5}\right)$ and $10 \mathrm{~g} .1^{-1}\left(\mathrm{Li}_{10}\right)$ and garlic extract at four levels, $0\left(\mathrm{Ga}_{0}\right), 5 \mathrm{ml} . \mathrm{l}^{-1}\left(\mathrm{Ga}_{5}\right), 10 \mathrm{ml} . \mathrm{l}^{-1}\left(\mathrm{Ga}_{10}\right)$ and $20 \mathrm{ml}^{-1} \mathrm{l}^{-1}\left(\mathrm{Ga}_{20}\right)$ and the interaction between them on leaves mineral and hormonal content. Each treatment was replicated three times with a factorial experiment using RCBD. 96 transplants were distributed among 16 treatments, 3 replicates each and 2 transplants in each experimental unite. Results of these experiments revealed that, the interaction between garlic and licorice root extracts significantly affected all the studied parameters. The highest means of most parameters were gained by the treatment of foliar spray with garlic extract at $20 \mathrm{ml}^{-1} \mathrm{l}^{-1}$ plus 5 or $10 \mathrm{gm} . \mathrm{l}^{-1}$ of licorice root extract. The foliar spray of $20 \mathrm{ml}$ garlic extract $+5 \mathrm{gm} \mathrm{.1} \mathrm{l}^{-1}$ licorice root extract gave the highest means of leaf area, while the highest means of leaf number, increased in main stem diameter, leaf chlorophyll content, were recorded for the treatment of $20 \mathrm{ml}$ garlic extract. $\mathrm{l}^{-1}+10$ gm licorice root extract $\mathrm{l}^{-1}$, respectively.
\end{abstract}

Key words: Garlic extract, licorice root extract, spray levels, vegetative growth, pear transplants. 